\title{
Assessing the Response of Sesame to Inorganic and Organic Nutrient Sources
}

\author{
Paul Anguria ${ }^{1}$, George N. Chemining'wa ${ }^{2}$, Richard N. Onwonga ${ }^{3} \&$ Michael A. Ugen $^{1}$ \\ ${ }^{1}$ National Semi Arid Resources Research Institute, Soroti, Uganda \\ ${ }^{2}$ Department of Plant Science and Crop Protection, University of Nairobi, Nairobi, Kenya \\ ${ }^{3}$ Department of Land Resources Management and Agricultural Technology, University of Nairobi, Nairobi, \\ Kenya \\ Correspondence: Paul Anguria, National Semi Arid Resources Research Institute, Serere, P.O. Box 56, Soroti, \\ Uganda. E-mail: paulanguria@yahoo.com
}

Received: July 19, 2019

Accepted: September 3, 2019 Online Published: December 15, 2019

doi:10.5539/jas.v12n1p108

URL: https://doi.org/10.5539/jas.v12n1p108

\begin{abstract}
Sesame (Sesamum indicum. L) is one of the main sources of livelihoods in northern Uganda. However, its production is constrained by low soil fertility and moisture levels. A study was conducted at Serere, Uganda in 2013 and 2014 to investigate the effect of organo-mineral fertilizers on growth, seed yield and nutritional quality of sesame. The design of the experiment was a randomized complete block design with three replications. The treatments comprised: control (no soil amendment), mixtures of 4 crop residues each at ( 3 and $6 \mathrm{t} / \mathrm{ha}$ ) and two rates of N, P and K. Finger millet husks ( $3 \mathrm{t} / \mathrm{ha})$ plus lower fertilizer rate $(30 \mathrm{~kg} \mathrm{~N}-25 \mathrm{~kg} \mathrm{P}-40 \mathrm{Kg} \mathrm{K} / \mathrm{ha})$ had significantly higher seed yield of sesame; while finger millet husks ( $6 \mathrm{t} / \mathrm{ha})$ plus higher fertilizer rate $(60 \mathrm{~kg} \mathrm{~N}-50$ $\mathrm{kg} \mathrm{P}-80 \mathrm{Kg} \mathrm{K} / \mathrm{ha}$ ) significantly increased vegetative growth of sesame. Finger millet husks (6 t/ha) plus lower fertilizer rate had significantly higher seed crude protein content of sesame; while cowpea husks ( $3 \mathrm{t} / \mathrm{ha}$ ) plus higher fertilizer rate and groundnut shells $(3 \mathrm{t} / \mathrm{ha}$ ) plus lower fertilizer rate produced significantly higher seed total ash and seed oil content of sesame, respectively. This study has demonstrated that application of a mixture of crop residues and inorganic fertilizers is the best treatment in enhancing growth, seed yield and nutritional seed quality of sesame.
\end{abstract}

Keywords: nutritional seed quality, seed crude protein and organo-mineral fertilizers

\section{Introduction}

Sesame (Sesamum indicum L.) is one of the most important oilseed crops in Uganda and is ranked second after groundnuts. Sesame seeds are used in different forms and constitute a major source of quality edible oil or oily paste, calcium and proteins (Anyanga et al., 2001; Bedigian, 2003). The residual meal after oil extraction constitutes a good source of proteins and $\mathrm{Ca}$ in livestock feeds (Munyua et al., 2013).

Sesame cultivation is confined mainly to drier and warmer regions of Uganda that receive very low and un-predictable rainfall. In addition, these areas have poor soils with low moisture retention and nutrient status. These soils are particularly low in N, P and K that are essential for sesame growth (Tenywa et al., 1999). Farmers in sesame growing areas are resource poor and heavily depend on rain-fed crop production systems and natural soil fertility for crop production (Ibeawuchi et al., 2009). Consequently, these farmers persistently get low sesame yields at their fields averaging $693 \mathrm{~kg} / \mathrm{ha}$ compared to a potential yield of $2250 \mathrm{~kg} / \mathrm{ha}$ (Anyanga et al., 2003; Munyua et al., 2013). This means farmers cannot accumulate sufficient funds to buy and use entirely inorganic fertilizers in sesame production. However, large quantities of crop residues are produced in farmers' fields that are usually burnt to clear fields for the next crop. The fact that crop residues are rich in organic matter and plant nutrients implies that they can enhance soil fertility and productivity of sesame when incorporated into the soil (Sanchez, 1992). But low biochemical quality of some crop residues reduces their nutrient release (Kriauciuniene et al., 2012). Enhancement of nutrient release from such crop residues can be achieved when applied in combination with $\mathrm{N}, \mathrm{P}$ and $\mathrm{K}$ nutrients.

Therefore, combined application of crop residues with inorganic fertilizers would most likely enhance sesame yield and nutritional quality, but also reduce the cost of using inorganic fertilizers singly. However, there is 
limited information on the effect of combined application of crop residues and $\mathrm{N}, \mathrm{P}$ and $\mathrm{K}$ nutrients on sesame yield and nutritional quality. A study was therefore conducted to assess the effect of crop residues and $\mathrm{N}, \mathrm{P}$ and $\mathrm{K}$ nutrients on seed yield and nutritional quality of sesame.

\section{Materials and Methods}

\subsection{Description of Study Site}

\subsubsection{Weather and Altitude}

This study was conducted at the National Semi-Arid Resources Research Institute (NaSARRI)-Serere, Uganda. NaSARRI is located at $0^{\circ} 32^{\prime} \mathrm{N}$ and $35^{\circ} 27^{\prime} \mathrm{E}$ at 1128 meters above sea level. The site experiences average annual temperatures of between $17.9{ }^{\circ} \mathrm{C}$ and $29.4{ }^{\circ} \mathrm{C}$. It receives an average annual rainfall of $1000 \mathrm{~mm}$ with a bimodal distribution. The relative humidity ranges between $72 \%$ and $84 \%$. January, February, June and July are the driest months with very little precipitation. However, the months of March, May, August and November are generally the wettest.

\subsubsection{Soils}

The soils at the experimental site are mainly sandy with low organic matter content and are classified as petricplinthosols although traces of soils classified as gleysols and vertisols also exist (Aniku, 2001). The soils at the experimental site are predominantly sandy silt loams with a $\mathrm{pH}$ ranging between 5.10 and 5.40 compared to the critical pH of 5.50. Calcium level $(3.13 \mathrm{me} / 100 \mathrm{~g})$ is low compared with the critical value of $4 \mathrm{me} / 100 \mathrm{~g}$. The amount of organic carbon is between 1.8 and $2.3 \%$ compared to the critical value of $6 \%$. These soils are also low in $\mathrm{P}(9.74$ $\mathrm{ppm})$ and $\mathrm{Na}(0.48 \mathrm{me} / 100 \mathrm{~g})$ compared to critical values of $15 \mathrm{ppm}$ and $1.01 \mathrm{me} / 100 \mathrm{~g}$, respectively. However, these soils are quite high in $\mathrm{Mg}(0.71 \mathrm{me} / 100 \mathrm{~g})$ and $\mathrm{K}(0.67 \mathrm{me} / 100 \mathrm{~g})$ compared with critical values of $0.50 \mathrm{me} / 100 \mathrm{~g}$ and $0.20 \mathrm{me} / 100 \mathrm{~g}$, respectively (Heckman, 1914; Mehlich, 1984; Lory et al., 2003).

\subsection{Treatments, Experimental Design and Crop Husbandry}

The design of this trial was a randomized complete block design with three replications. The treatments comprised of two rates ( 3 and $6 \mathrm{t} / \mathrm{ha}$ ) of crop residues (husks of millet, sorghum, cowpea and groundnuts) and two rates of NPK nutrients (30 kg N-25 kg P-40 kg K/ha) and (60 kg N-50 kg P-80 kg K/ha). Crop residues were analyzed for chemical elements before they were applied on experimental plots. Each experimental plot measured $4 \mathrm{~m} \times 4 \mathrm{~m}$ with a path of $1 \mathrm{~m}$ separating plots. Sesame variety Sesim II was used in this study. It is highly branching with an average height of $100 \mathrm{~cm}$ and a maturity period of 90 days. Six seeds were planted per hill at inter and intra row spacing of $30 \mathrm{~cm}$ and $15 \mathrm{~cm}$, respectively. The crop was then thinned to one plant per hill at three weeks after planting. The crop was hand weeded three times before it was harvested.

\subsection{Data recording}

\subsubsection{Plant Growth Parameters}

Sesame plant growth parameters measured included: plant height, number of branches per plant and leaf area index (LAI). Ten sesame plants at physiological maturity were selected randomly from each plot and their heights measured with a meter rod and number of branches counted. The LAI was determined using a leaf canopy analyzer-Licor-2010 at 75\% flowering stage. Five plants were measured per plot.

\subsubsection{Sesame Seed Yield}

Seed weight per plant, 1000 seed weight and seed yield were determined. Seed weight per plant was determined by weighing seeds from capsules of 10 plants. A thousand seed weight was determined by getting three sets of 1000 seeds from each seed lot derived from individual plots and weighing each of the sets. Seed yield was determined by threshing sun dried sesame plants from each plot, cleaning them and weighing the resultant seeds using a weighing scale.

\subsubsection{Sesame Seed Nutritional Quality}

Seed oil content, crude protein content and total ash content of sesame were determined. Seed oil content was determined using the Soxhlet method (AOAC, 1990). Crude Protein and total ash content in sesame seeds were determined using the Kjeldahl and dry ashing methods respectively. The conversion factor of 6.25 was used to convert Kjeldahl nitrogen to (\%) protein (Nelson et al., 2010).

\subsection{Data Analysis}

Recorded data were subjected to analysis of variance (ANOVA) using Genstat statistical package $13^{\text {th }}$ edition, 2013. The means were compared using the least significant difference test (LSD) at $\mathrm{p} \leq 0.05$. 


\section{Results}

\subsection{Effect of Crop Residues and Inorganic Fertilizers on Plant Height, Number of Branches, and Leaf Area Index} of Sesame

Incorporation of groundnut shells and husks of finger millet and cowpea in soil produced significantly $(\mathrm{P} \leq 0.05)$ taller sesame plants $(104.7$ to $163.1 \mathrm{~cm})$ than NPK fertilizer and the control that had a height range $(98.7$ to 140 $\mathrm{cm}$ ) (Table 1). In contrast, application of $6 \mathrm{t} / \mathrm{ha}$ of sorghum husks produced shorter sesame plants (77.3-100.7 $\mathrm{cm}$ ) than NPK fertilizer, and the control which had a height range $(98.7$ to $140 \mathrm{~cm}$ ) (Table 1). Application of 60 $\mathrm{kg} \mathrm{N}-50 \mathrm{~kg} \mathrm{P}-80 \mathrm{~kg} \mathrm{~K} / \mathrm{ha}$ (higher fertilizer rate) produced taller sesame plants with a higher number of branches per plant and LAI compared with $30 \mathrm{~kg} \mathrm{~N}-25 \mathrm{~kg}$ P-40 kg K/ha (lower fertilizer rate) (Table 1). However, combined application of 6 t/ha of finger millet husks and $60 \mathrm{~kg} \mathrm{~N}-50 \mathrm{~kg} \mathrm{P}-80 \mathrm{~kg} \mathrm{~K} / \mathrm{ha}$ produced significantly P $\leq$ 0.05 taller sesame plants with higher number of branches per plant and LAI than all other treatments (Tables 1, 2 and 3). Sesame plants were also significantly $(\mathrm{P} \leq 0.05)$ taller where sorghum were applied in combination with $60 \mathrm{~kg} \mathrm{~N}-50 \mathrm{~kg} \mathrm{P}-80 \mathrm{~kg} \mathrm{~K} / \mathrm{ha}$ than the control and sorghum husks plus $30 \mathrm{~kg} \mathrm{~N}-25 \mathrm{~kg}$ P-40 kg K/ha (Tables 2 and $3)$.

Table 1. Effect of crop residues and inorganic fertilizer on plant height, no of branches/plant and leaf area index of sesame

\begin{tabular}{|c|c|c|c|c|c|c|c|c|c|}
\hline \multirow{2}{*}{ Treatments } & \multicolumn{3}{|c|}{ Plant height (cm) } & \multicolumn{3}{|c|}{ No of branches/plant } & \multicolumn{3}{|c|}{ Leaf area index } \\
\hline & 2013SR & 2014LR & 2014SR & 2013SR & 2014LR & 2014 SR & 2013SR & 2014LR & 2014 SR \\
\hline Control & 111.5 & 98.7 & 117.2 & 2.03 & 2.13 & 3.63 & 2.04 & 1.80 & 2.68 \\
\hline \multicolumn{10}{|l|}{ Crop residues } \\
\hline Millet husks $3 \mathrm{t} / \mathrm{ha}$ & 126.9 & 105.7 & 130.3 & 4.30 & 2.33 & 4.20 & 2.79 & 2.89 & 4.22 \\
\hline Millet husks 6 t/ha & 135.1 & 116.5 & 163.1 & 4.48 & 2.73 & 5.27 & 3.24 & 4.41 & 4.27 \\
\hline Sorghum husks $3 \mathrm{t} / \mathrm{ha}$ & 110.7 & 89.1 & 111.9 & 1.86 & 2.10 & 3.60 & 1.99 & 1.70 & 2.12 \\
\hline Sorghum husks $6 \mathrm{t} / \mathrm{ha}$ & 90.1 & 77.3 & 100.7 & 1.64 & 1.60 & 3.00 & 1.94 & 1.19 & 1.75 \\
\hline Cowpea husks 3 t/ha & 126.9 & 105.1 & 141.4 & 3.73 & 1.80 & 3.78 & 2.96 & 3.50 & 3.70 \\
\hline Cowpea husks $6 \mathrm{t} / \mathrm{ha}$ & 129.5 & 108.4 & 147.5 & 4.46 & 2.47 & 5.20 & 3.11 & 3.85 & 4.23 \\
\hline Groundnut shells $3 \mathrm{t} / \mathrm{ha}$ & 129.9 & 104.7 & 125.6 & 3.21 & 2.17 & 3.74 & 2.77 & 3.22 & 3.60 \\
\hline Groundnut shells 6 t/ha & 130.9 & 106.6 & 128.1 & 4.27 & 2.73 & 3.80 & 3.09 & 3.72 & 3.65 \\
\hline \multicolumn{10}{|l|}{ NPK fertilizer } \\
\hline $\mathrm{NPK}_{1}(30 \mathrm{~kg} \mathrm{~N}-25 \mathrm{~kg} \mathrm{P}-40 \mathrm{~kg} \mathrm{~K} / \mathrm{ha})$ & 131.5 & 106.1 & 134.3 & 2.28 & 2.33 & 4.80 & 2.61 & 4.05 & 4.33 \\
\hline $\mathrm{NPK}_{2}(60 \mathrm{~kg} \mathrm{~N}-50 \mathrm{~kg} \mathrm{P}-80 \mathrm{~kg} \mathrm{~K} / \mathrm{ha})$ & 132.7 & 123.7 & 140.0 & 3.16 & 3.33 & 6.20 & 3.00 & 6.21 & 4.37 \\
\hline P-value & 0.003 & 0.003 & 0.001 & 0.02 & 0.03 & 0.04 & 0.03 & 0.02 & 0.001 \\
\hline LSD $(P<0.05)$ & 13.3 & 16.9 & 15.1 & 1.51 & 1.72 & 1.91 & 1.56 & 2.53 & 0.92 \\
\hline
\end{tabular}

Note. Control $=$ no amendment, $\mathrm{LR}=$ long rains, $\mathrm{SR}=$ short rains, $\mathrm{LAI}=$ leaf area index and no $=$ number.

Table 2. Effect of crop residues combined with $\mathrm{NPK}_{1}$ fertilizer on plant height, no of branches/plant and leaf area index of sesame

\begin{tabular}{|c|c|c|c|c|c|c|c|c|c|}
\hline \multirow{2}{*}{ Treatments } & \multicolumn{3}{|c|}{ Plant height (cm) } & \multicolumn{3}{|c|}{ No of branches/plant } & \multicolumn{3}{|c|}{ Leaf area index } \\
\hline & 2013SR & 2014LR & 2.014SR & 2013SR & 2014LR & 2014SR & 2013SR & 2014LR & 2014SR \\
\hline Control & 111.5 & 98.7 & 117.2 & 2.03 & 2.13 & 3.63 & 2.04 & 1.80 & 2.68 \\
\hline \multicolumn{10}{|c|}{ Crop residues plus $\mathrm{NPK}_{1}$ fertilizer } \\
\hline Millet (3 t/ha) plus $\mathrm{NPK}_{1}$ & 127.9 & 121.1 & 126.4 & 2.37 & 2.40 & 5.20 & 2.51 & 5.11 & 3.53 \\
\hline Millet (6 t/ha) plus NPK $_{1}$ & 131.9 & 140.5 & 181.9 & 2.81 & 3.80 & 5.67 & 2.84 & 5.29 & 4.52 \\
\hline Sorghum ( $3 \mathrm{t} / \mathrm{ha})$ plus $\mathrm{NPK}_{1}$ & 109.4 & 113.9 & 131.8 & 2.80 & 2.67 & 5.47 & 2.96 & 5.58 & 3.37 \\
\hline Sorghum (6 t/ha) plus $\mathrm{NPK}_{1}$ & 98.1 & 81.3 & 101.3 & 2.84 & 3.00 & 5.53 & 3.14 & 7.30 & 3.77 \\
\hline Cowpea (3t/ha) plus $\mathrm{NPK}_{1}$ & 127.0 & 129.8 & 132.0 & 1.47 & 2.73 & 4.27 & 5.49 & 7.96 & 5.57 \\
\hline Cowpea (6 t/ha) plus $\mathrm{NPK}_{1}$ & 128.8 & 134.5 & 135.3 & 2.58 & 3.33 & 5.07 & 3.00 & 4.94 & 4.22 \\
\hline Groundnut ( $3 \mathrm{t} / \mathrm{ha}$ ) plus $\mathrm{NPK}_{1}$ & 119.5 & 122.2 & 132.5 & 2.47 & 2.67 & 4.67 & 2.17 & 4.25 & 3.63 \\
\hline Groundnut $(6 \mathrm{t} / \mathrm{ha})$ plus $\mathrm{NPK}_{1}$ & 126.4 & 123.9 & 135.9 & 2.69 & 2.93 & 5.17 & 3.60 & 4.83 & 4.25 \\
\hline P-value & 0.003 & 0.003 & 0.001 & 0.02 & 0.03 & 0.04 & 0.03 & 0.02 & 0.001 \\
\hline LSD $(\mathrm{P}<0.05)$ & 13.3 & 16.9 & 15.1 & 1.51 & 1.72 & 1.91 & 1.56 & 2.53 & 0.92 \\
\hline
\end{tabular}

Note. Control $=$ no amendment, $\mathrm{SR}=$ short rains, $\mathrm{LR}=$ long rains, $\mathrm{NPK}_{1}=30 \mathrm{~kg} \mathrm{~N}-25 \mathrm{~kg} \mathrm{P}-40 \mathrm{~kg} \mathrm{~K} / \mathrm{ha}$ and no $=$ number. 
Table 3. Effect of crop residues combined with $\mathrm{NPK}_{2}$ fertilizer on plant height, no of branches/plant and leaf area index of sesame

\begin{tabular}{|c|c|c|c|c|c|c|c|c|c|}
\hline \multirow{2}{*}{ Treatments } & \multicolumn{3}{|c|}{ Plant height (cm) } & \multicolumn{3}{|c|}{ No of branches/plant } & \multicolumn{3}{|c|}{ Leaf area index } \\
\hline & 2013SR & 2014LR & 2.014SR & 2013SR & 2014LR & 2014SR & 2013SR & 2014LR & 2014SR \\
\hline Control & 111.5 & 98.7 & 117.2 & 2.03 & 2.13 & 3.63 & 2.04 & 1.80 & 2.68 \\
\hline \multicolumn{10}{|c|}{ Crop residues plus $\mathrm{NPK}_{2}$ fertilizer } \\
\hline Millet (3 t/ha) plus $\mathrm{NPK}_{2}$ & 129.1 & 118.2 & 133.5 & 2.17 & 2.47 & 5.87 & 2.10 & 5.23 & 3.57 \\
\hline Millet (6 t/ha) plus $\mathrm{NPK}_{2}$ & 137.5 & 148.4 & 182.5 & 4.14 & 4.20 & 6.13 & 5.47 & 7.99 & 4.75 \\
\hline Sorghum ( $3 \mathrm{t} / \mathrm{ha})$ plus $\mathrm{NPK}_{2}$ & 124.9 & 136.9 & 126.2 & 3.29 & 3.20 & 5.40 & 2.70 & 4.73 & 4.00 \\
\hline Sorghum ( $6 \mathrm{t} / \mathrm{ha})$ plus $\mathrm{NPK}_{2}$ & 128.5 & 141.9 & 137.0 & 3.75 & 4.00 & 4.47 & 3.61 & 6.67 & 4.23 \\
\hline Cowpea (3 t/ha) plusNPK 2 & 124.8 & 135.6 & 135.9 & 2.35 & 2.47 & 5.33 & 4.13 & 4.47 & 4.77 \\
\hline Cowpea (6 t/ha) plus $\mathrm{NPK}_{2}$ & 134.6 & 141.2 & 160.2 & 4.10 & 4.00 & 5.93 & 2.93 & 4.89 & 4.03 \\
\hline Groundnut (3t/ha) plus $\mathrm{NPK}_{2}$ & 121.9 & 131.9 & 126.1 & 2.39 & 2.60 & 4.47 & 3.14 & 4.03 & 3.67 \\
\hline Groundnut $(6 \mathrm{t} / \mathrm{ha})$ plus $\mathrm{NPK}_{2}$ & 128.7 & 136.3 & 137.7 & 2.77 & 2.93 & 5.67 & 2.57 & 4.17 & 3.90 \\
\hline P-value & 0.003 & 0.003 & 0.001 & 0.02 & 0.03 & 0.04 & 0.03 & 0.02 & 0.001 \\
\hline LSD $(P<0.05)$ & 13.3 & 16.9 & 15.1 & 1.51 & 1.72 & 1.91 & 1.56 & 2.53 & 0.92 \\
\hline
\end{tabular}

Note. Control $=$ no amendment, $\mathrm{LR}=$ long rains, $\mathrm{SR}=$ short rains, $\mathrm{NPK}_{2}=60 \mathrm{~kg} \mathrm{~N}-50 \mathrm{~kg} \mathrm{P}-80 \mathrm{~kg} \mathrm{~K} / \mathrm{ha}$ and no $=$ number.

\subsection{Effect of Crop Residues and Inorganic Fertilizers on Seed Yield of Sesame}

Application of $3 \mathrm{t} / \mathrm{ha}$ of finger millet husks produced significantly $(\mathrm{P} \leq 0.05)$ a higher sesame seed yield $(0.50$ to $1.95 \mathrm{t} / \mathrm{ha})$ compared to inorganic fertilizer which produced sesame seed yield $(0.33$ to $1.41 \mathrm{t} / \mathrm{ha})$ (Table 5). In contrast, incorporation of $6 \mathrm{t} / \mathrm{ha}$ of sorghum husks produced a lower seed yield of sesame than inorganic fertilizer, $3 \mathrm{t} / \mathrm{ha}$ of sorghum husks and other crop residues (Table 5). Plots treated with $30 \mathrm{~kg} \mathrm{~N}-25 \mathrm{~kg} \mathrm{P}-40 \mathrm{~kg}$ $\mathrm{K} /$ ha (lower fertilizer rate) had a significantly $(\mathrm{P} \leq 0.05)$ higher seed yield of sesame than plots amended with 60 $\mathrm{kg} \mathrm{N}-50 \mathrm{~kg} \mathrm{P}-80 \mathrm{~kg} \mathrm{~K} / \mathrm{ha}$ (higher fertilizer rate) (Table 5). While application of $3 \mathrm{t} / \mathrm{ha}$ of finger millet husks plus $30 \mathrm{~kg} \mathrm{~N}-25 \mathrm{~kg} \mathrm{P}-40 \mathrm{~kg} \mathrm{~K} / \mathrm{ha}$ produced significantly $(\mathrm{P} \leq 0.05)$ higher seed yield of sesame than all other treatments (Table 6).

Table 5. Effect of crop residues, inorganic fertilizers and their combination on sesame seed yield

\begin{tabular}{|c|c|c|c|c|c|c|c|c|c|}
\hline \multirow{2}{*}{ Treatments } & \multicolumn{3}{|c|}{ Seed wt per plant (g) } & \multicolumn{3}{|c|}{1000 seed wt (g) } & \multicolumn{3}{|c|}{ Seed yield (t/ha) } \\
\hline & 2013SR & 2014LR & 2014SR & 2013SR & 2014LR & 2014SR & 2013SR & 2014LR & 2014SR \\
\hline Control & 1.99 & 4.54 & 12.4 & 2.45 & 3.07 & 5.42 & 0.32 & 0.46 & 0.76 \\
\hline \multicolumn{10}{|l|}{ Crop residues } \\
\hline Millet (3 t/ha) & 2.84 & 8.78 & 19.3 & 3.49 & 5.58 & 9.53 & 0.50 & 0.53 & 1.95 \\
\hline Millet (6 t/ha) & 2.27 & 5.06 & 17.0 & 2.22 & 4.59 & 8.35 & 0.33 & 0.24 & 1.89 \\
\hline Sorghum (3 t/ha) & 1.17 & 4.38 & 16.3 & 2.00 & 3.01 & 4.17 & 0.14 & 0.33 & 0.62 \\
\hline Sorghum (6 t/ha) & 1.09 & 3.97 & 16.4 & 1.94 & 2.54 & 4.08 & 0.12 & 0.30 & 0.56 \\
\hline Cowpea (3 t/ha) & 2.01 & 8.28 & 18.0 & 2.97 & 5.31 & 9.08 & 0.49 & 0.45 & 1.94 \\
\hline Cowpea (6 t/ha) & 2.06 & 4.77 & 18.0 & 2.47 & 3.34 & 8.19 & 0.23 & 0.44 & 1.90 \\
\hline Groundnut (3t/ha) & 2.05 & 5.62 & 11.0 & 2.53 & 2.78 & 5.55 & 0.23 & 0.43 & 0.99 \\
\hline Groundnut ( $6 \mathrm{t} / \mathrm{ha})$ & 2.20 & 5.92 & 16.3 & 2.54 & 4.41 & 5.58 & 0.34 & 0.51 & 1.29 \\
\hline \multicolumn{10}{|l|}{ Inorganic fertilizer } \\
\hline $\mathrm{NPK}_{1}$ & 3.86 & 8.81 & 17.5 & 2.85 & 5.02 & 6.88 & 0.34 & 0.89 & 1.61 \\
\hline $\mathrm{NPK}_{2}$ & 2.24 & 6.50 & 12.8 & 2.20 & 4.73 & 4.56 & 0.33 & 0.71 & 1.41 \\
\hline P-value & 0.013 & 0.04 & 0.09 & 0.001 & 0.011 & 0.02 & 0.001 & 0.007 & 0.007 \\
\hline LSD (5\%) & 1.79 & 4.20 & NS & 0.17 & 0.48 & 0.27 & 0.16 & 0.47 & 0.53 \\
\hline
\end{tabular}

Note. Control $=$ no amendment, $\mathrm{NS}=$ not significant, $\mathrm{SR}=$ short rains, $\mathrm{LR}=$ long rains and $\mathrm{NPK}_{2}=60 \mathrm{~kg} \mathrm{~N}-50$ $\mathrm{kg} \mathrm{P}-80 \mathrm{~kg} \mathrm{~K} / \mathrm{ha}$. 
Table 6. Effect of crop residues combined with $\mathrm{NPK}_{1}$ fertilizer on sesame seed yield

\begin{tabular}{|c|c|c|c|c|c|c|c|c|c|}
\hline \multirow{2}{*}{ Treatments } & \multicolumn{3}{|c|}{ Seed weight per plant (g) } & \multicolumn{3}{|c|}{1000 seed weight $(\mathrm{g})$} & \multicolumn{3}{|c|}{ Seed yield (t/ha) } \\
\hline & 2013SR & 2014LR & 2014SR & 2013SR & 2014LR & 2014SR & 2013SR & 2014LR & 2014SR \\
\hline Control & 1.19 & 4.54 & 12.4 & 2.45 & 3.07 & 5.42 & 0.32 & 0.46 & 0.76 \\
\hline \multicolumn{10}{|c|}{ Crop residues plus $N P K_{1}$ fertilizer } \\
\hline Millet (3 $\mathrm{t} / \mathrm{ha}$ ) plus $\mathrm{NPK}_{1}$ & 4.10 & 9.33 & 19.8 & 3.56 & 6.91 & 9.88 & 0.50 & 0.96 & 1.94 \\
\hline Millet (6 t/ha) plus $\mathrm{NPK}_{1}$ & 3.04 & 8.40 & 14.8 & 3.24 & 3.69 & 7.52 & 0.34 & 0.90 & 1.75 \\
\hline $\operatorname{Sorghum}(3 \mathrm{t} / \mathrm{ha})$ plus $\mathrm{NPK}_{1}$ & 1.23 & 3.21 & 14.0 & 2.51 & 3.46 & 5.69 & 0.34 & 0.86 & 1.57 \\
\hline Sorghum (6 t/ha) plus $\mathrm{NPK}_{1}$ & 0.83 & 2.92 & 16.0 & 2.45 & 3.51 & 5.54 & 0.34 & 0.79 & 1.74 \\
\hline Cowpea (3 t/ha) plus NPK 1 & 3.85 & 8.75 & 16.7 & 2.98 & 4.05 & 7.75 & 0.81 & 0.94 & 1.79 \\
\hline Cowpea (6 t/ha) plus NPK 1 & 2.80 & 8.61 & 14.5 & 2.53 & 3.66 & 7.33 & 0.75 & 0.76 & 1.53 \\
\hline Groundnut ( $3 \mathrm{t} / \mathrm{ha}$ ) plus $\mathrm{NPK}_{1}$ & 3.00 & 4.78 & 13.8 & 2.47 & 3.46 & 7.18 & 0.51 & 0.67 & 1.54 \\
\hline Groundnut ( $6 \mathrm{t} / \mathrm{ha}$ ) plus $\mathrm{NPK}_{1}$ & 2.69 & 5.82 & 9.0 & 2.53 & 3.48 & 5.31 & 0.41 & 0.83 & 1.62 \\
\hline P-value & 0.013 & 0.04 & 0.09 & 0.001 & 0.011 & 0.02 & 0.001 & 0.007 & 0.007 \\
\hline LSD $(\mathrm{P}<0.05)$ & 1.79 & 4.20 & NS & 0.17 & 0.48 & 0.27 & 0.16 & 0.47 & 0.53 \\
\hline
\end{tabular}

Note. Control $=$ no amendment, $\mathrm{NPK}_{1}=30 \mathrm{~kg} \mathrm{~N}-25 \mathrm{~kg} \mathrm{P}-40 \mathrm{~kg} \mathrm{~K} / \mathrm{ha}, \mathrm{NS}=$ not significant, SR = short rains and $\mathrm{LR}=$ long rains.

Table 7. Effect of crop residues combined with $\mathrm{NPK}_{2}$ fertilizer on sesame seed yield

\begin{tabular}{|c|c|c|c|c|c|c|c|c|c|}
\hline \multirow{2}{*}{ Treatments } & \multicolumn{3}{|c|}{ Seed weight per plant (g) } & \multicolumn{3}{|c|}{1000 seed weight (g) } & \multicolumn{3}{|c|}{ Seed yield (t/ha) } \\
\hline & 2013SR & 2014LR & 2014SR & 2013SR & 2014LR & 2014 SR & 2013SR & 2014LR & 2014SR \\
\hline Control & 1.99 & 4.54 & 12.4 & 2.45 & 3.07 & 5.42 & 0.32 & 0.46 & 0.76 \\
\hline \multicolumn{10}{|c|}{ Crop residues plus $\mathrm{NPK}_{2}$ fertilizer } \\
\hline Millet (3 t/ha) plus $\mathrm{NPK}_{2}$ & 3.74 & 8.41 & 16.0 & 3.07 & 5.00 & 7.00 & 0.59 & 0.82 & 1.90 \\
\hline Millet (6 t/ha) plus $\mathrm{NPK}_{2}$ & 2.46 & 5.80 & 10.0 & 2.47 & 2.17 & 6.06 & 0.26 & 0.90 & 1.60 \\
\hline Sorghum ( $3 \mathrm{t} / \mathrm{ha}$ ) plus $\mathrm{NPK}_{2}$ & 2.92 & 6.96 & 13.0 & 2.54 & 4.90 & 5.50 & 0.26 & 0.83 & 1.41 \\
\hline Sorghum (6 t/ha) plus $\mathrm{NPK}_{2}$ & 2.09 & 7.14 & 13.1 & 2.56 & 2.78 & 5.49 & 0.37 & 0.73 & 1.33 \\
\hline Cowpea (3t/ha) plus $\mathrm{NPK}_{2}$ & 3.31 & 9.11 & 15.8 & 2.63 & 3.56 & 6.07 & 0.49 & 0.82 & 1.82 \\
\hline Cowpea (6 t/ha) plus $\mathrm{NPK}_{2}$ & 2.82 & 8.21 & 12.7 & 2.45 & 3.42 & 5.50 & 0.32 & 0.88 & 1.69 \\
\hline Groundnut ( $3 \mathrm{t} / \mathrm{ha}$ ) plusNPK 2 & 2.82 & 6.19 & 10.7 & 2.58 & 3.54 & 4.32 & 0.40 & 0.77 & 1.18 \\
\hline Groundnut ( $6 \mathrm{t} / \mathrm{ha}$ ) plus $\mathrm{NPK}_{2}$ & 3.30 & 7.93 & 16.1 & 2.60 & 3.51 & 4.93 & 0.44 & 0.71 & 1.07 \\
\hline P-value & 0.013 & 0.04 & 0.09 & 0.001 & 0.011 & 0.02 & 0.001 & 0.007 & 0.007 \\
\hline $\operatorname{LSD}(\mathrm{P}<0.05)$ & 1.79 & 4.20 & NS & 0.17 & 0.48 & 0.27 & 0.16 & 0.47 & 0.53 \\
\hline
\end{tabular}

Note. Control $=$ no amendment, $\mathrm{NS}=$ not significant, $\mathrm{SR}=$ short rains, $\mathrm{LR}=$ long rains and $\mathrm{NPK}_{2}=60 \mathrm{~kg} \mathrm{~N}-50$ $\mathrm{kg} \mathrm{P}-80 \mathrm{~kg} \mathrm{~K} / \mathrm{ha}$.

\subsection{Effect of Crop Residues and Inorganic Fertilizers on Seed Crude Protein, Total Ash and Oil Contents of Sesame}

Incorporation of $6 \mathrm{t} / \mathrm{ha}$ of finger millet husks into soil had significantly $(\mathrm{P} \leq 0.05)$ higher sesame seed crude protein content than finger millet husks ( $3 \mathrm{t} / \mathrm{ha}$ ) and other crop residues (3.1). Plots treated with $60 \mathrm{~kg} \mathrm{~N}-50 \mathrm{~kg}$ $\mathrm{P}-80 \mathrm{~kg} \mathrm{~K} / \mathrm{ha}$ (higher fertilizer rate) had significantly $(\mathrm{P} \leq 0.05)$ higher sesame seed crude protein and total ash contents than $30 \mathrm{~kg} \mathrm{~N}-25 \mathrm{~kg} \mathrm{P}-40 \mathrm{~kg} \mathrm{~K} / \mathrm{ha}$ (lower fertilizer rate) (Table 8). Application of $6 \mathrm{t} / \mathrm{ha}$ of finger millet husks plus $30 \mathrm{~kg} \mathrm{~N}-25 \mathrm{~kg} \mathrm{P}-40 \mathrm{~kg} \mathrm{~K} / \mathrm{ha}, 3 \mathrm{t} / \mathrm{ha}$ of cowpea husks plus $60 \mathrm{~kg} \mathrm{~N}-50 \mathrm{~kg} \mathrm{P}-80 \mathrm{~kg} \mathrm{~K} / \mathrm{ha}$ and $3 \mathrm{t} / \mathrm{ha}$ of groundnut shells plus $30 \mathrm{~kg} \mathrm{~N}-25 \mathrm{~kg} \mathrm{P}-40 \mathrm{~kg} \mathrm{~K} / \mathrm{ha}$ produced significantly $(\mathrm{P} \leq 0.05)$ higher sesame seed crude protein, total ash and oil content than other treatments (Tables 9 and 10). Single application of higher fertilizer rate and crop residues significantly $(\mathrm{P} \leq 0.05)$ decreased sesame seed oil content. For instance application $6 \mathrm{t} / \mathrm{ha}$ of finger millet husks decreased sesame seed oil content from 28.55 to $22.86 \%$ in 2014 long rains (Table 8). 
Table 8. Effect of crop residues and inorganic fertilizers on seed crude protein, total ash and oil content of sesame

\begin{tabular}{|c|c|c|c|c|c|c|c|c|c|}
\hline \multirow{2}{*}{ Treatments } & \multicolumn{3}{|c|}{ Crude protein (\%) } & \multicolumn{3}{|c|}{ Total ash (\%) } & \multicolumn{3}{|c|}{ Oil content (\%) } \\
\hline & 2013SR & 2014LR & 2014SR & 2013LR & 2014LR & 2014SR & 2013SR & 2014LR & 2014SR \\
\hline Control & 24.10 & 23.13 & 21.14 & 3.36 & 4.12 & 4.57 & 22.12 & 22.86 & 24.49 \\
\hline \multicolumn{10}{|l|}{ Crop residues } \\
\hline Millet (3 t/ha) & 27.17 & 26.71 & 23.64 & 5.02 & 4.54 & 6.90 & 27.89 & 30.85 & 26.12 \\
\hline Millet (6 t/ha) & 27.80 & 28.18 & 28.28 & 4.67 & 4.24 & 6.24 & 25.62 & 28.55 & 26.53 \\
\hline Sorghum (3t/ha) & 25.18 & 25.80 & 21.18 & 4.44 & 6.07 & 7.42 & 22.28 & 24.70 & 24.51 \\
\hline Sorghum (6 t/ha) & 26.49 & 27.57 & 22.61 & 4.46 & 4.98 & 6.44 & 27.55 & 30.52 & 25.78 \\
\hline Cowpea (3 t/ha) & 25.39 & 26.69 & 22.98 & 4.44 & 4.26 & 6.10 & 30.69 & 33.62 & 31.60 \\
\hline Cowpea (6 t/ha) & 27.43 & 27.88 & 23.89 & 4.89 & 6.13 & 7.76 & 26.91 & 29.33 & 25.44 \\
\hline Groundnut (3t/ha) & 24.19 & 25.74 & 19.38 & 3.68 & 5.52 & 6.05 & 32.43 & 35.56 & 33.34 \\
\hline Groundnut ( $6 \mathrm{t} / \mathrm{ha})$ & 27.13 & 27.43 & 21.18 & 4.13 & 5.96 & 7.66 & 40.04 & 43.51 & 40.35 \\
\hline \multicolumn{10}{|l|}{ NPK fertilizer } \\
\hline $\mathrm{NPK}_{1}$ & 26.53 & 26.67 & 22.65 & 4.23 & 4.05 & 6.43 & 32.24 & 35.20 & 30.47 \\
\hline $\mathrm{NPK}_{2}$ & 26.61 & 27.09 & 23.91 & 4.28 & 5.94 & 7.47 & 20.31 & 22.98 & 20.74 \\
\hline P-value & 0.001 & 0.004 & 0.002 & 0.001 & 0.19 & 0.003 & 0.001 & 0.001 & 0.001 \\
\hline LSD $(\mathrm{P}<0.05)$ & 0.58 & 0.76 & 1.63 & 0.66 & NS & 0.97 & 0.46 & 1.38 & 0.92 \\
\hline
\end{tabular}

Note. Control $=$ no amendment, $\mathrm{NS}=$ not significant, $\mathrm{LR}=$ long rains, $\mathrm{SR}=$ short rains, $\mathrm{NPK}_{1}=30 \mathrm{~kg} \mathrm{~N}-25 \mathrm{~kg}$ $\mathrm{P}-40 \mathrm{~kg} \mathrm{~K} / \mathrm{ha}$ and $60 \mathrm{~kg} \mathrm{~N}-50 \mathrm{~kg} \mathrm{P}-80 \mathrm{~kg} \mathrm{~K} / \mathrm{ha}$ ).

Table 9. Effect of crop residues combined with $\mathrm{NPK}_{1}$ fertilizer on seed crude protein, total ash and oil content of sesame

\begin{tabular}{|c|c|c|c|c|c|c|c|c|c|}
\hline \multirow{2}{*}{ Treatments } & \multicolumn{3}{|c|}{ Crude protein $(\%)$} & \multicolumn{3}{|c|}{ Total ash $(\%)$} & \multicolumn{3}{|c|}{ Oil content $(\%)$} \\
\hline & 2013SR & 2014LR & 2014SR & 2013SR & 2014LR & 2014SR & 2013SR & 2014LR & 2014SR \\
\hline Control & 24.10 & 23.13 & 21.14 & 3.36 & 4.12 & 4.57 & 22.12 & 22.86 & 24.49 \\
\hline \multicolumn{10}{|c|}{ Crop residues plus $\mathrm{NPK}_{1}$ fertilizer } \\
\hline Millet (3 t/ha) plus $\mathrm{NPK}_{1}$ & 27.17 & 26.37 & 24.18 & 4.16 & 5.12 & 5.88 & 36.55 & 39.51 & 34.78 \\
\hline Millet (6 t/ha) plus $\mathrm{NPK}_{1}$ & 28.42 & 28.49 & 26.07 & 4.60 & 4.40 & 6.25 & 25.04 & 27.46 & 25.95 \\
\hline Sorghum ( $3 \mathrm{t} / \mathrm{ha})$ plus $\mathrm{NPK}_{1}$ & 25.58 & 25.14 & 20.04 & 4.06 & 5.52 & 5.83 & 32.61 & 35.03 & 32.53 \\
\hline Sorghum (6t/ha) plus $\mathrm{NPK}_{1}$ & 25.34 & 26.55 & 22.53 & 4.09 & 6.00 & 6.71 & 28.28 & 31.21 & 29.19 \\
\hline Cowpea ( $3 \mathrm{t} / \mathrm{ha})$ plus $\mathrm{NPK}_{1}$ & 28.32 & 26.39 & 22.80 & 4.44 & 4.32 & 6.81 & 36.69 & 40.91 & 37.61 \\
\hline Cowpea (6 t/ha) plusNPK 1 & 27.70 & 27.77 & 24.45 & 4.43 & 4.25 & 6.72 & 31.62 & 34.55 & 30.84 \\
\hline Groundnut (3t/ha) plus $\mathrm{NPK}_{1}$ & 25.71 & 25.74 & 21.28 & 4.38 & 5.82 & 6.62 & 38.49 & 44.65 & 37.92 \\
\hline Groundnut $(6 \mathrm{t} / \mathrm{ha})$ plus $\mathrm{NPK}_{1}$ & 26.49 & 25.41 & 20.31 & 4.32 & 4.20 & 6.67 & 30.13 & 33.09 & 28.36 \\
\hline P-value & 0.001 & 0.004 & 0.002 & 0.001 & 0.19 & 0.003 & 0.001 & 0.001 & 0.001 \\
\hline LSD $(\mathrm{P}<0.05)$ & 0.58 & 0.76 & 1.63 & 0.66 & NS & 0.97 & 0.46 & 1.38 & 0.92 \\
\hline
\end{tabular}

Note. Control $=$ no amendment, NS $=$ not significant, $\mathrm{NPK}_{1}=30 \mathrm{~kg} \mathrm{~N}-25 \mathrm{~kg} \mathrm{P}-40 \mathrm{~kg} \mathrm{~K} / \mathrm{ha}, \mathrm{SR}=$ short rains and $\mathrm{LR}=$ long rains. 
Table 10. Effect of crop residues combined with $\mathrm{NPK}_{2}$ fertilizer on seed crude protein, total ash and oil content of sesame

\begin{tabular}{|c|c|c|c|c|c|c|c|c|c|}
\hline \multirow{2}{*}{ Treatments } & \multicolumn{3}{|c|}{ Crude protein (\%) } & \multicolumn{3}{|c|}{ Total ash (\%) } & \multicolumn{3}{|c|}{ Oil content $(\%)$} \\
\hline & 2013SR & 2014LR & 2014SR & 2013SR & 2014LR & 2014SR & 2013SR & 2014LR & 2014SR \\
\hline Control & 24.10 & 23.13 & 21.14 & 3.36 & 4.12 & 4.57 & 22.12 & 22.86 & 24.49 \\
\hline \multicolumn{10}{|l|}{ Crop residuesplus $\mathrm{NPK}_{2}$ fertilizer } \\
\hline Millet (3 t/ha) plus $\mathrm{NPK}_{2}$ & 27.13 & 27.17 & 23.33 & 4.60 & 4.33 & 6.34 & 20.43 & 21.39 & 19.88 \\
\hline Millet (6 t/ha) plus $\mathrm{NPK}_{2}$ & 27.43 & 27.25 & 24.17 & 4.77 & 6.17 & 6.86 & 20.46 & $21 . .34$ & 13.64 \\
\hline Sorghum ( $3 \mathrm{t} / \mathrm{ha})$ plus $\mathrm{NPK}_{2}$ & 25.26 & 24.22 & 19.32 & 4.65 & 4.36 & 6.07 & 27.27 & 31.06 & 26.53 \\
\hline Sorghum ( $6 \mathrm{t} / \mathrm{ha})$ plus $\mathrm{NPK}_{2}$ & 25.59 & 25.46 & 19.99 & 4.72 & 5.62 & 6.24 & 28.30 & 28.86 & 18.58 \\
\hline Cowpea (3t/ha) plus $\mathrm{NPK}_{2}$ & 26.12 & 26.15 & 22.88 & 4.58 & 5.81 & 8.03 & 24.92 & 21.88 & 21.34 \\
\hline Cowpea (6 t/ha) plus $\mathrm{NPK}_{2}$ & 27.19 & 27.26 & 24.07 & 4.36 & 4.24 & 5.75 & 22.43 & 23.36 & 24.58 \\
\hline Groundnut ( $3 \mathrm{t} / \mathrm{ha})$ plus $\mathrm{NPK}_{2}$ & 25.96 & 26.00 & 21.77 & 3.91 & 5.34 & 6.97 & 29.11 & 31.53 & 30.02 \\
\hline Groundnut ( $6 \mathrm{t} / \mathrm{ha})$ plus $\mathrm{NPK}_{2}$ & 25.59 & 25.33 & 21.16 & 4.16 & 5.67 & 7.31 & 28.97 & 30.23 & 25.50 \\
\hline P-value & 0.001 & 0.004 & 0.002 & 0.001 & 0.19 & 0.003 & 0.001 & 0.001 & 0.001 \\
\hline LSD $(P<0.05)$ & 0.58 & 0.76 & 1.63 & 0.66 & NS & 0.97 & 0.46 & 1.38 & 0.92 \\
\hline
\end{tabular}

Note. Control $=$ no amendment, $\mathrm{NS}=$ not significant, $\mathrm{NPK}_{2}=60 \mathrm{~kg} \mathrm{~N}-50 \mathrm{~kg} \mathrm{P}-80 \mathrm{~kg} \mathrm{~K} / \mathrm{ha}, \mathrm{SR}=$ short rains and $\mathrm{LR}=$ long rains.

\section{Discussion}

The development of significantly taller plants in plots generally treated with groundnut shells and husks of finger millet and cowpea could be attributable to higher quantities of organic $\mathrm{C}, \mathrm{N}$ and other nutrients added into the soil upon their decomposition. This could have enhanced soil moisture retention and nutrient uptake by sesame resulting in enhanced vegetative growth. Rawls et al. (2003) similarly reported that increase in levels of organic $\mathrm{C}$ in soil correspondingly increases soil moisture retention and nutrient uptake. In contrast, shorter sesame plants produced in plots treated with $6 \mathrm{t} / \mathrm{ha}$ of sorghum husks could be attributable to increased levels of sorgoleone supplied by sorghum husks that inhibited sesame growth. Khaliq et al. (2011) similarly observed negative shoot growth of horse purslane crop treated with sorghum straw. They equally attributed it to the presence of sorgoleone in sorghum straw. But in plots treated with a mixture of higher fertilizer rate and sorghum husks, taller sesame crops were observed. This could be attributed to high amount of $\mathrm{N}$ supplied by higher fertilizer rate that could have enhanced mineralization of sorghum husks resulting in reduced phytotoxic effects of sorgoleone on sesame. Eviner et al. (2008) similarly reported that manipulating $\mathrm{C} / \mathrm{N}$ ratio of allelopathic plant residues to enhance their mineralization reduces their phytotoxic effects on other crop species. The excessive sesame vegetative growth observed where $60 \mathrm{~kg} \mathrm{~N}-50 \mathrm{~kg} \mathrm{P}-80 \mathrm{~kg} \mathrm{~K} / \mathrm{ha}$ was applied singly, and a mixture of 6 tons of finger millet plus higher fertilizer rate was applied could be attributed to higher amounts of $\mathrm{P}, \mathrm{K}$ and more especially $\mathrm{N}$ added into the soil. This most likely caused enhanced vegetative growth of sesame. Kashani et al. (2015) similarly reported that application of $\mathrm{N}$ in the range of 70 to $112.5 \mathrm{~kg} / \mathrm{ha}$ and $\mathrm{P}$ in the range of 45 to 70 $\mathrm{kg} / \mathrm{ha}$ significantly increased vegetative growth of sesame. In addition, finger millet husks may have also increased soil moisture retention that promoted vegetative growth of sesame. Jassimuddin (2014) also reported that application of higher NPK fertilizer rate plus mustard oil cake produced higher vegetative growth of sesame plants. This was equally attributed to improved soil moisture retention and increased supply of N, P and K nutrients.

Production of significantly higher sesame seed yield in plots treated with $3 \mathrm{t} / \mathrm{ha}$ of finger millet husks than inorganic fertilizer and other crop residues could be due to organic $\mathrm{C}$ and adequate quantities of $\mathrm{N}, \mathrm{P}$ and $\mathrm{K}$ nutrients added into the soil by this treatment. Consequently, this most likely enhanced soil moisture retention and nutrient uptake by sesame that could have in turn promoted development of reproductive structures. Ebaid (2007) similarly reported that application of rice husks in soil increased sesame water use efficiency and seed yield. This was attributed to improvement of soil moisture retention and improved nutrient supply. However, reduced sesame seed yield due to application of $6 \mathrm{t} / \mathrm{ha}$ of sorghum husks could be due to large quantities of sorgoleone added into the soil that adversely affected the growth and development of reproductive structures of sesame compared to other treatments (Westen et al., 2013). Significantly higher sesame seed yield observed in plots treated with lower fertilizer rate than higher fertilizer rate could be due to addition of optimal quantities of $\mathrm{N}, \mathrm{P}$ and $\mathrm{K}$ into the soil that may have promoted development of reproductive structures. Thakur et al. (2004) and Shehu et al. (2014) also reported that application of $20 \mathrm{~kg} \mathrm{~N} / \mathrm{ha}, 24.9 \mathrm{~kg}$ to $37.3 \mathrm{~kg} \mathrm{~K} / \mathrm{ha}$ and $22.5 \mathrm{~kg} \mathrm{P} / \mathrm{ha}$ significantly increased sesame seed yield. This was attributed to optimum supply of N, P and $\mathrm{K}$ which promoted 
development of sesame reproductive structures. Significantly higher sesame seed yields observed in plots treated with $3 \mathrm{t} / \mathrm{ha}$ of finger millet husks plus lower fertilizer rate compared to other treatments, could be attributable to addition of sufficient quantities of organic $\mathrm{C}, \mathrm{N}, \mathrm{P}, \mathrm{K}$ and $\mathrm{Ca}$ and enhanced soil moisture retention by finger millet husks (Anguria et al., 2017). In addition, lower fertilizer rate might have also added into soil optimum quantities of N, P and K nutrients. The above conditions could have been favored development of reproductive structures. Soliman et al. (2014) similarly reported that application of organic manures in combination with inorganic fertilizers increases crop yields because of enhanced $\mathrm{N}$ use efficiency and improvement in soil moisture retention.

Significantly higher sesame seed crude protein produced in plots treated with 6 t/ha of finger millet, higher fertilizer rate and a mixture of $6 \mathrm{t} / \mathrm{ha}$ of finger millet husks plus lower fertilizer rate is attributable to high $\mathrm{N}$ added into the soil (Anguria et al., 2017). High N supply could have enhanced its uptake that most likely promoted protein synthesis in sesame seeds. Khaliq (2004) also reported that incorporation of high levels of organic and inorganic $\mathrm{N}$ fertilizers into the soil could have been responsible for increased sesame seed crude protein content. This was equally attributed to enhanced protein synthesis by N. However, higher seed oil content observed in plots treated with lower fertilizer rate could be attributed to optimum $\mathrm{P}$ and $\mathrm{K}$ supply that most likely promoted lipid and carbohydrate metabolism in sesame seeds that resulted in enhanced oil synthesis. Hafiz et al. (2012) similarly reported that oil content of sesame increased with application of 45 to $95 \mathrm{~kg} \mathrm{P} / \mathrm{ha}$. This was also attributed to promotion of lipid metabolism by P which increased seed oil content (Ali et al., 2002). Generally, the highest sesame seed oil content observed in plots treated with a mixture of $3 \mathrm{t} / \mathrm{ha}$ of groundnut shells plus lower fertilizer rate compared with other treatments could be due to addition of optimum levels of N, P and K. Consequently, it most likely promoted carbohydrate metabolism which enhanced oil synthesis in sesame seeds. In the contrary, significantly reduced sesame seed oil content observed in plots treated with higher fertilizer rate and crop residues may be attributed to increased $\mathrm{N}$ levels that might have inhibited lipid and carbohydrate metabolism. Studies by Tahir et al. (2003) also indicate that higher rates of N significantly reduced sesame seed oil contents due to its negative effect on carbohydrate metabolism. Similarly, Zhao et al. (1993) and Asare et al. (1995) reported that increased applications of $\mathrm{N}$ fertilizer on canola significantly decreased its oil content. But in contrast they observed that reduced levels of $\mathrm{N}$ fertilizer had no effect on oil content of canola seeds. Lastly, significantly higher levels of total ash observed in sesame seeds in plots treated with $3 \mathrm{t} / \mathrm{ha}$ of cowpea husks plus $60 \mathrm{~kg} \mathrm{~N}-50 \mathrm{~kg} \mathrm{P}-80 \mathrm{~kg} \mathrm{~K} / \mathrm{ha}$ could be due to high levels of N, P and K nutrients added into the soil. This could have enhanced their uptake by sesame leading to subsequent accumulation in seeds.

\section{Conclusions}

Generally, the highest plant growth, seed yield and nutritional quality of sesame occurred in plots treated with crop residues than inorganic fertilizer. Single application of finger millet husks produced the highest sesame growth and seed yield than other crop residues. Application of mixtures of $3 \mathrm{t} / \mathrm{ha}$ of finger millet, $6 \mathrm{t} / \mathrm{ha}$ of finger millet husks, $3 \mathrm{t} / \mathrm{ha}$ of cowpea husks and $3 \mathrm{t} / \mathrm{ha}$ of groundnut shells with lower fertilizer rate produced the highest seed yield, seed crude protein content, seed total ash content, seed oil content and seed yield of sesame, respectively, compared to other treatments. Sole application of sorghum husks suppressed growth and yield of sesame, while combined application of sorghum husks with inorganic fertilizer enhanced growth and yield of sesame. Finger millet husks are more effective in enhancing protein levels in sesame seeds. While combined application of crop residues and inorganic fertilizer is more effective in improving nutritional seed quality of sesame than sole application of crop residues and inorganic fertilizer.

\section{References}

Ali, E. A., \& Sakr, M. M. (2002). Effect of plant spacing and phosphorus fertilization on anatomical structure, yield and yield components of sesame (Sesamum indicum L.) plant. The 3rd Scientific Conference of Agricultural Science, Assiut.

Anguria, P., Chemining'wa, G. N., Onwonga, R. N., \& Ugen, M. A. (2017). Decomposition and Nutrient Release of Selected Cereal and Legume Crop Residues. Journal of Agricultural Science, 9(6), 108. https://doi.org/ 10.5539/jas.v9n6p108

Aniku, J. R. F. (2001). Soil classification and pedology. Kampala, Uganda: Fountain Publishers.

Anyanga, W. O., \& Obong, Y. Y. (2001). Sesame (Sesamum indicum L.) In J. K. Mukiibi (Ed.), Agriculture in Uganda, 11, 97-102.

Anyanga, W. O., Obong, Y., Busolo-Bulafu, C. M., ZudeErobot C., Odul, C. S., Oumo, J. R., ... Elayu, M. (2003). A decade of sesame breeding in Uganda. Ugandan Journal of Agricultural Sciences, 8, 135-140. 
AOAC (Association of Official Analytical Chemists). (1990). Methods of the Association of Official Analytical Chemists (Method No. 920.85, 15th ed., p. 780). AOAC, Arlington, VA, USA.

Asare, E., \& Scarisbrick, D. H. (1995). Rate of nitrogen and sulfur fertilizers on yield, yield components and seed quality of oilseed rape (Brassica napus L.). Field Crops Resources, 44, 41-46. https://doi.org/10.1016/ 0378-4290(95)00051-7

Bedigian, D., \& van der Maesen, L. J. G. (2003). Slimy Leaves and Oily Seeds: Distribution and Use of Sesamum spp. and Ceratotheca sesamoides (Pedaliaceae) in Africa (pp. 271-274).

Ebaid, A. (2007). Exact solitary wave solutions for some nonlinear evolution equations via Exp-function method. Physics Letters A, 365(3), 213-219. https://doi.org/10.1016/j.physleta.2007.01.009

Eviner, V. T., \& Hawkes, C. V. (2008). Embracing variability in the application of plant-soil interactions to the restoration of communities and ecosystems. Restoration Ecology, 137(16), 713-729. https://doi.org/10.1111/ j.1526-100X.2008.00482.x

Hafiz, S. I., \& El-Bramawy, M. A. S. (2012). Response of sesame (Sesamum indicum L.) to phosphorus fertilization and spraying with potassium in newly reclaimed sandy soils. International Journal of Agricultural Science Research, 1(3), 034-040.

Heckman, J. R. (1914). Soil fertility test interpretation phosphorus, potassium, magnesium, and calcium. Extension specialist in soil fertility. The State University of New Jersey, USA.

Ibeawuchi, I. I., Obiefuna, J. C., Ofor, M. O., Ihem, E. E., Nwosu, F. O., Nkwocha, V. I., ... Phelps, S. (2013). Effects of nitrogen fertilizer application on seed yield, $\mathrm{N}$ uptake, $\mathrm{N}$ use efficiency, and seed quality of Brassica carinata. Canadian Journal of Plant Science, 93(6), 1073-1081. https://doi.org/10.4141/ cjps2013-222

Jasimuddin, S. K. (2014). Effect of integrated nutrient management practices in summer sesame. Retrieved from https://www.blogger.com

Kashani, S., Buriro, M., Nadeem, A., Ahmed, N., Saeed, Z., Mohammad, F., \& Ahmed, S. (2015). Response of various sesame varieties under the influence of Nitrogen and phosphorus doses. American Journal of Plant Sciences, 6, 405-412. https://doi.org/10.4236/ajps.2015.62046

Khaliq, A. (2004). Irrigation and nitrogen management effects on productivity of hybrid sunflower (Helianthus annuus L.) $\mathrm{PhD}$ Thesis submitted to the department of agronomy, University of Agricultural Sciences, Faisalabad, Pakistan.

Khaliq, A., Matloob, A., Farooq, M., Mushtaq, M. N., \& Khan, M. B. (2011). Effect of crop residues applied isolated or in combination on the germination and seedling growth of horse purslane (Trianthema portulacastrum). Planta Daninha, 29(1), 121-128. https://doi.org/10.1590/S0100-83582011000100014

Kriauciuniene, Z., Velička, R., \& Raudonius, S. (2012). The influence of crop residues type on their decomposition rate in the soil: a litter bag study. Zemdirbyste Agriculture, 99(3), 227-236.

Lory, J., \& Scharf, P. (2003). Soil Specific Phosphorus and Potassium Recommendations-Critical Values. Department of Agronomy, University of Missouri.

Mehlich, A. (1984). Mehlich-3 soil test extractant: A modification of Mehlich-2 extractant. Commun. Soil Sci. Plant Anal., 15(12), 1409-1416. https://doi.org/10.1080/00103628409367568

Munyua, B., Orr, A., \& Okwadi, J. (2013). Open sesame: A value chain analysis of Sesame Marketing in Northern Uganda. Socioeconomics discussion paper series (Series paper number 6).

Nanjundappa, G., Shivaraj, B., Janarjuna, S., \& Sridhara, S. (2001). Effect of organic and inorganic sources of nutrients applied alone or in combination on growth and yield of sunlower (Helianthus annuus L.). Helia, 24(34), 115-120. https://doi.org/10.1515/helia.2001.24.34.115

Nelson, D. W., \& Sommers, L. E. (2010). Methods of soil analysis. Retrieved November 15, 2010, http://www.aaslpsu.edu/soils.methodshtm

Rawls, W. J., Pachepsky, Y. A., Ritchie, J. C., Sobecki, T. M., \& Bloodworth, H. (2003). Effect of soil organic carbon on soil water retention. Geoderma, 116, 61-76. https://doi.org/10.1016/S0016-7061(03)00094-6

Shehu, H. E. (2014). Uptake and agronomic efficiencies of nitrogen, phosphorus and potassium in sesame (Sesamum indicum L.). American Journal of Plant Nutrition and Fertilization Technology, 4, 41-56. https://doi.org/10.3923/ajpnft.2014.41.56 
Soliman, S. M., Ghabour, S., Galal, Y. G. M., \& EL-Sofi, D. M. (2014). Alternative strategies for improving nitrogen nutrition of some economical crops using $15 \mathrm{~N}$ stable isotope. International Journal of Current Microbiology and Applied Science, 3(7), 970-983.

Tahir, M., Asghar Malik, M., Tanveer, A., \& Ahmad, R. (2003). Effect of NPK Levels on Seed Yield and Oil Contents of Canola. Pak. J. Life Soc. Sci., 1(2), 127-132.

Tenywa, J. S., Kidoido, M., Nyende, P., Kasenge, V., Oryokot, J., \& Mbowa, S. (1999). Prospects and constraints of finger millet production in Eastern Uganda. African Crop Science Journal, 7, 563-578. https://doi.org/ 10.4314/acsj.v7i4.27751

Thakur, D. S., \& Patel, S. R. (2004). Response of sesame (Sesamum indicum) to different levels of potassium and sulphur in light-textured Inceptisols of eastern part of Chhattisgarh. Indian Journal of Agriculture Science, 74, 496-498.

Weston, L. A., Alsaadawi, I. S., \& Baerson, S. R. (2013). Sorghum allelopathy from ecosystem to molecule. Journal of Chemical Ecology, 39(2), 142-53. https://doi.org/10.1007/s10886-013-0245-8

Yin, X., \& Vyn, T. J. (2003). Potassium placement effects on yield and seed composition of no-till soybean seed in alternate row widths. Agron J., 95, 126-132. https://doi.org/10.2134/agronj2003.0126

Zhao, F., Evans, J., Bilsborrow, P. E., \& Syers, J. K. (1993). Influence of sulphur and nitrogen on seed yield and quality of low glucosinolate oilseed rape (Brassica napus L.). Journal of Science for Food and Agriculture, 6391, 29-37. https://doi.org/10.1002/jsfa.2740630106

\section{Copyrights}

Copyright for this article is retained by the author(s), with first publication rights granted to the journal.

This is an open-access article distributed under the terms and conditions of the Creative Commons Attribution license (http://creativecommons.org/licenses/by/4.0/). 